\title{
Ebola Viral Hemorrhagic Disease Outbreak in West Africa- Lessons from Uganda.
}

\author{
Anthony K. Mbonye*1, Joseph F. Wamala ${ }^{2}$, Miriam Nanyunja ${ }^{3}$, Alex Opio ${ }^{4}$, \\ Issa Makumbi ${ }^{5}$, Jane Ruth Aceng ${ }^{6}$
}

1. Associate Professor, School of Public Health- Makerere University \&, Commissioner Health Services, Ministry of Health, Box 7272, Kampala, Uganda

2. Senior Epidemiologist, Epidemiology and Surveillance Division, Ministry of Health, Box 7272, Kampala, Uganda

3. Disease Prevention and Control Officer, WHO Uganda Country Office, P. O. Box 24578, Kampala.

4. Assistant Commissioner Health Services, Ministry of Health, Box 7272, Kampala, Uganda.

5. Epidemiology and Surveillance Division, Ministry of Health, Box 7272, Kampala, Uganda

6. Director General of health services, Ministry of Health, Box 7272, Kampala, Uganda

\begin{abstract}
Background: There has been a rapid spread of Ebola Viral Hemorrhagic disease in Guinea, Liberia and Sierra Leone since March 2014. Since this is the first time of a major Ebola outbreak in West Africa; it is possible there is lack of understanding of the epidemic in the communities, lack of experience among the health workers to manage the cases and limited capacities for rapid response. The main objective of this article is to share Uganda's experience in controlling similar Ebola outbreaks and to suggest some lessons that could inform the control of the Ebola outbreak in West Africa.

Methods: The article is based on published papers, reports of previous Ebola outbreaks, response plans and experiences of individuals who have participated in the control of Ebola epidemics in Uganda.

Lessons learnt: The success in the control of Ebola epidemics in Uganda has been due to high political support, effective coordination through national and district task forces. In addition there has been active surveillance, strong community mobilization using village health teams and other community resources persons, an efficient laboratory system that has capacity to provide timely results. These have coupled with effective case management and infection control and the involvement of development partners who commit resources with shared responsibility.
\end{abstract}

Conclusion: Several factors have contributed to the successful quick containment of Ebola outbreaks in Uganda. West African countries experiencing Ebola outbreaks could draw some lessons from the Uganda experience and adapt them to contain the Ebola epidemic.

Key words: Ebola, viral hemorrhagic fever, West Africa, lessons, Uganda

DOI: http/dx.doi.org/10.4314/ahs.v14i3.1

\section{Background}

On 23th March, 2014, the Ministry of Health of Guinea notified the World Health Organisation of the outbreak of Ebola Virus Disease1. The cases of Ebola were initially reported from three southeastern districts of Gueckedou, Macenta, Nzerekore, and Kissidougou in the forest region; and in Conakry, the capital city. The tests on the samples taken from suspected patients were positive for Ebola Zaire, thus confirming the first Ebola outbreak in Guinea1.

\section{*correspondence author:}

Anthony K. Mbonye

email: ak mbonye@yahoo.com /vpad

mn@infocom,co.oug;

Tel: +256-772411668 retrospective epidemiologic investigation indicated that the first suspect case in the outbreak2 was a 2-year-old child from Meliandou, Gueckedou that died on 6 December 2013. Between December 2013 and March 2014, the outbreak spread from Gueckedou, to Macenta, Kissidougou, and Conakry, the capital city2, 3. The Ebola outbreak then spread to the neighbouring countries of Liberia and Sierra Leone. The Ministry of Health and Social Welfare of Liberia formally declared Ebola outbreak on 29th March, 2014 while Sierra Leone notified the first case of Ebola to the WHO on 25 May, 2014 4,5. As of 17th July, 2014, the cumulative number of cases attributed to Ebola in the three countries was 1,048 and 632 deaths (Case Fatality Rate of 60\%) 6.

The transmission of Ebola in W. Africa has been char- 
acterized by the following four major patterns: 6

-Transmission dominantly in the rural communities

-Transmission mainly in the densely populated periurban cities of Conakry and Monrovia

-Transmission along the shared border areas of Guinea, Liberia and Sierra Leone.

-Persistent transmission in health care settings with several health workers contacting and dying from Ebola virus disease.

In this paper, we share Uganda's experiences in controlling similar Ebola outbreaks, and suggest some best practices that could inform the control of the Ebola outbreak in West Africa, and perhaps address the different challenges being faced in its containment.

Methods:

The article is based on review of published papers and updates on the Ebola outbreak in West-Africa, published papers about the previous Viral Hemorrhagic Fever outbreaks in Uganda, reports of previous Ebola outbreaks, response plans and experiences of individuals who have participated in the control of Ebola epidemics in Uganda. The authors were all actively involved in the response to Viral Hemorrhagic Fever outbreaks in Uganda.

The Major Challenges of the Ebola Outbreak in W. Africa:

-There is lack of understanding of the epidemic in the communities ("there is no such thing as Ebola" says the community), lack of experience among the health workers to manage the cases and limited capacities for rapid response as this is the first Ebola outbreak in West Africa.

-High exposure to the Ebola virus in the communities through household care and customary burial procedures e.g. use bare hands to wash dead bodies. This has resulted into high number of deaths in the communities leading to panic and anxiety. There are misconceptions on the new disease; and this has led to denials, mistrust, hostility to health workers especially foreign health workers and rejection of public health interventions. There are beliefs and myths that impede acceptance of messages about treatment; for example communities believe hospitalization is a death sentence.

-The other challenge relates to the fact that by the time the outbreak was finally confirmed and declared in Guinea on 21 March 2014, several clusters of cases, suspected to have started several months back were identified in hospitals in Gueckedou and Macenta 2, 7. Due to the delayed detection of Ebola virus disease in Guinea, transmission chains accumulated leading to increased number of Ebola cases that were dispersed over wide geographical areas2, 7. It therefore became an immense and complicated challenge to trace all these chains of transmission dispersed over wide geographical locations by teams of public health workers that have no previous experience in responding to Ebola virus disease outbreaks.

-Emergency response relies a lot on the capacity of the prevailing health systems before the disaster strikes. It is now well established that just like the other counties where Ebola has caused outbreaks in sub-Saharan Africa in the past, the three counties affected by the current Ebola outbreak in West Africa have weak health systems with acute shortages of the much needed human resources and lack of in-country capacities to confirm Filoviruses such as Ebola 7, 8. The delayed detection of cases at the onset of outbreaks is a major indicator of the level of functionality of the health system and the corresponding surveillance systems for detecting priority diseases.

-The persistent number of deaths among health workers shows that the three counties lacked preparedness for Ebola virus disease outbreaks. Consequently, the healthcare workers lacked training on infection control precautions including barrier nursing that are requisite for healthcare worker safety so that the much needed patient care is provided 8 .

-Current information from the outbreaks indicates that Ebola cases were either missed by active case finding teams or were actually withdrawn from the isolation facilities by relatives even after they were confirmed to have Ebola 8. This breach of infection control and isolation recommendations can only worsen outbreak trends as it actually did in West Africa. It has been revealed that the decision by relatives to withdraw patients confirmed to have Ebola from the isolation facilities was prompted by the decision to transfer cases to distant hospitals 8 . Transfer of Ebola patients over long distances is never a good idea due to the risk of spreading the outbreak to new locations and the risk of losing the much-needed cooperation of the affected communities. The fact that response teams were eventually turned away from affected communities shows that this trust and confidence had been breached.

\section{Uganda's Experience in Controlling Ebola and Other Epidemics}

Over the last 15 years, Uganda has had several viral hemorrhagic fever (VHF) outbreaks 9-21. The first and biggest Ebola outbreak in Uganda occurred in 2000 with cases being reported in the three districts of Gulu, 
Masindi, and Mbarara 9. In the subsequent years, outbreaks were reported in Bundibugyo, Luwero, Kibaale, and Luwero districts in 2007, 2011, 2012 and 2012 respectively 10-13. Marburg virus disease outbreaks were also reported in Ibanda and Kamwenge in 2007; involving cases in the Netherlands and United States respectively that were exposed after visiting caves in Bushenyi district; and in 2012 with cases confirmed in Ibanda and Kabale districts 13,14. A major outbreak of Yellow Fever was reported in five districts in Northern Uganda in 2010 with 181 cases including 45 deaths being reported 10.

In virtually all the above, the disease outbreaks were controlled with limited spread of the epidemics much beyond the initial loci of the outbreak. In addition, the case fatality rates for Ebola were Gulu [52.7\%]; Bundibugyo [32\%]; Luwero 2011 [(1/1) -100\%]; Kibaale [70.8\%]; and Luwero [57.1\%] 15.

Thus Uganda has accumulated enough experience that can be shared with other countries that face the risk of these epidemics. Specifically, the following strategies were used:

-Uganda has standing multi-sectoral and multidisciplinary task force committees on epidemics that include partners and NGOs at the national and district level. The national task force is composed of experts (epidemiologists, laboratory scientists, communication experts, psychiatrists and psychologists, physicians, veterinarians, etc.) from the Ministry of Health, Ministry of Agriculture, Office of the Prime Minister and partners including WHO, CDC, UNICEF, AFENET, Uganda Red Cross, and MSF and it meets monthly; however it meets daily when there is an epidemic.

Similarly, all the districts have task forces composed of the district political, civic, and health leadership as well as technical advisors from different partners working in the districts. Both the National and district task forces have subcommittees that are responsible for overseeing and implementing different components of epidemic response; the subcommittees include coordination, epidemiology and laboratory, case management, social mobilization, logistics, and psychosocial support and all have clear terms of reference. In addition, national rapid response teams and district rapid response teams are constituted immediately an epidemic is notified. They conduct investigations and support the establishment of an appropriate response in collaboration with the task forces. To reinforce response coordination at the district level, two or three senior officers are deployed from the national level to the district level to work with the district task force. There is daily contact between the district and national task forces during epidemic time. The Ministers of Health and other senior officers in the ministry of health and WHO visit the affected areas to explain the government actions, seek the community's cooperation, and motivate the response teams.

During the inter-epidemic periods, monthly taskforce meetings are convened to review disease surveillance data and update epidemic preparedness and response plans. There is constant communication between district surveillance officers and the national level especially the division of disease surveillance in the ministry of health.

- Uganda was one of the first countries in WHO-AFRO to adopt the integrated disease surveillance and response strategy (IDSR) in 2000. Over the years, the national surveillance system has been strengthened based on this strategy. The designated surveillance focal points at health sub-district, district, and regional levels, have been trained and facilitated to ensure timely detection, reporting, and investigation of priority diseases. These have been linked to the HMIS system for reporting of public health events of national and/or international concern. The village health teams (VHTs) upon hearing or detecting any reportable priority disease report to the nearest health facility. These are important links between the facility-based surveillance system and the community. The surveillance system is further enhanced during epidemics to enable daily tracing of all contacts of viral hemorrhagic fever patients and immediate investigation and isolation of suspected cases. This ensures that transmission within the community is controlled quickly. Uganda has recently established an emergency operations centre supported by CDC and this provides timely data and maps of epidemics.

- Uganda has overtime built a good laboratory network within districts, regional referral hospitals and at the national level. The samples for detection of Ebola are analyzed in Entebbe at the Uganda Virus Research Institute (UVRI). Uganda has also built capacity in specimen collection, processing, packaging and storage and an efficient specimen shipment mechanism.

-Uganda has developed local capacity for social mobilization during epidemics. We engage the political, local, opinion and religious eaders through meetings, radio messages and community meetings. Effort is made to first understand the knowledge, attitudes and beliefs of the affected people and this informs the social mobilization efforts. We work with partners including UNICEF and The Uganda Red Cross in this endeavor.

-Uganda has developed local experience in case management, infection prevention and control (IPC). 
We have trained many health workers on Viral Haemorrhagic Fevers (VHF) case management and other epidemics over the years. We have training guidelines that have been updated over time. The health workers are deployed to manage cases in collaboration with partners like MSF during the epidemics. Isolation facilities are set up in health facilities close to the affected areas to minimize transfer of the patients for long distances. A list of the trained and experienced health workers and their contacts is maintained at national level for quick contact when the health workers are needed in districts outside their usual work place.

-During the VHF epidemics psychosocial support to affected patients, their attendants, and communities has been adopted as a practice. A team of psychosocial support providers engages the affected communities to understand their misconceptions and misgivings, takes time to address them and discuss socially accepted solutions which are then adopted by the response team. The psychosocial team also counsels the patients and their immediate family about the natural progression of the disease and the expected outcomes; and keeps the immediate family briefed on the situation of the patient in isolation facility. In addition, the team provides psychosocial support to the health workers working in isolation facility to avoid burnout and depression.

-To further prevent transmission within the affected communities, the affected homes are disinfected and the contaminated personal effects of the patients disinfected and destroyed. The personal effects the patients come with to isolation facility are also disinfected and destroyed at discharge. To replace the destroyed personal effects, a "discharge package" is given to the patient on discharge and the family of the deceased patients. This enhances cooperation of the families with sick patients that need to be taken to the isolation facility.

-Close supervision of the response by the National Task Force, the top leadership of the Ministry of Health, and the top Government leadership.

-Uganda has been working with neighboring countries to strengthen the cross-border surveillance and management of epidemics. This should be strengthened among the W. African countries

Challenges Uganda has Met in Controlling Ebola Viral Hemorrhagic Disease

-The delayed detection of viral hemorrhagic fever outbreaks as we have seen in the West African Ebola outbreak has been a challenge in most of the Ebola outbreaks in Uganda. This challenge is attributed to the non-specific presentation of cases at the onset of Ebola virus disease outbreaks. To improve the detection of these atypical disease outbreaks the Uganda Ministry of Health has initiated the following interventions: Standardized clinical and community case definitions for all priority diseases including mysterious cases/deaths have been developed and disseminated to all health facilities and VHTs. In addition, village health teams (VHTs) have been trained on using the community case definitions to detect and report emerging disease outbreaks or mysterious diseases to the nearest health facilities for further verification. Also, as part of the nationwide drive by the Ministry of Health to strengthen the national disease surveillance system, district health teams and rapid response teams are being trained to enhance the implementation of the integrated disease surveillance and response strategy at all levels.

- Before the Ebola outbreak in Bundibugyo in 2007, Uganda lacked in-country capacities for laboratory confirmation of Ebola and other Filoviruses. This led to major delays of up to four weeks in securing laboratory confirmation. With support from the Centres for Disease Control and Prevention, a P3+ laboratory has been established at the UVRI and this has reduced the turnaround time to less than 24 hours. This has therefore been a major boost for response and case management teams to rollout timely outbreak response interventions following the confirmation of new Filovirus outbreaks.

- Dealing with Ebola cases that are dispersed over a wide geographic expanse is never an easy undertaking. One strategy that has worked for Uganda is to have a decentralized response with outbreak coordination committees formed in the respective districts that are affected by the outbreak. In addition, the decentralized district outbreak response teams have onsite support from senior technical officers from the national taskforce with experience in managing all the technical interventions for containing an Ebola outbreak. There is also remote support for the district outbreak response teams from the national taskforce through regular telephone conference calls, sharing of situation reports, and sharing minutes of the taskforce meetings.

-The need for strong and effective health systems is paramount for effective disease outbreak response. The need for adequate numbers of well-trained health care workers with adequate medicines, infection control supplies, infrastructure, and equipment for case referral and isolation is paramount. Thus Uganda has developed a roster of well-trained health workers with experience in managing Filovirus disease cases. These experts have been deployed to support response to Ebola outbreaks in Uganda and to all the three countries that are cur- 
rently affected by Ebola in West Africa. Additionally, the Uganda government has embarked on a phased plan of setting up isolation facilities at all major hospitals. During the first phase of this program, a state-ofthe-art isolation facility has been built in Entebbe with support from the World Bank. To enhance capacities for case detection and management of events due to especially dangerous pathogens (EDPs) like Filoviruses, the Uganda Ministry of Health with support from the Defense Threat Reduction Agency (DTRA) is conducting trainings for healthcare workers countrywide.

- Involving local leaders is a crucial component of ensuring community compliance to the recommended public health interventions for Ebola control. Thus district outbreak response teams are by default chaired by elected local leaders that have the full mandate of the affected communities. In addition, the Government of Uganda has rolled out a community network of Village Health Team members (VHTs). The VHTs are community health workers that are selected by the community to support the implementation of public health interventions at the community level. The VHTs have proved to be a vital resource for supporting outbreak response activities. During the past Ebola outbreaks, VHTs have been used to conduct door-to-door active case search, contact tracing and follow-up, and house-to-house health education on Ebola prevention and control. Since VHTs work in the very communities that elected them, they are more accepted and hence mistrust and rejection of response teams has not been reported during the recent Filovirus outbreaks in Uganda.

-A unique challenge that we have not had to deal with in the past but which is an issue during the current Ebola outbreaks in West Africa is containing transmission in closely knit cross-border communities. The newly established WHO centre for coordinating response to the outbreaks in the three countries should come up with a strategy for enhancing cross-border communications to facilitate follow-up of contacts, case isolation, and educating communities on Ebola prevention and control Uganda's response to the Ebola outbreak in W. Africa On 8th April, 2014, the Ministry of Health through its Public Emergency Operations Centre informed the districts on the progress of the Ebola outbreak in West Africa and urged health workers to have a high index of suspicion especially for travelers to and from West Africa.

-The Ministry of Health issued a public press release (4th July 2014) and advised the public on the necessary precautions. All district task forces were alerted.
-The Ministry has instituted measures for screening and follow- up of travelers from W. Africa at the Entebbe international airport.

-The National Task Force is operational; and The Rapid Response Teams (RRT) is on full alert and ready to take action when necessary.

\section{Lessons for West Africa}

Based on the above experience from Uganda, successful containment of VHF epidemics will depend on effective epidemic coordination at national and sub-national levels in the West-African affected countries. Functional national and sub-national epidemic management committees and response teams that sit daily (to coordinate, review challenges and come up with workable solutions) should be established and/or strengthened. In addition, the W. African countries should strengthen active surveillance, involve local community health workers and volunteers in active surveillance and contact tracing to ensure that all suspected cases are detected timely and removed from the community. Local capacity for case management should be urgently built and case management centres established close to the epidemic hot spots to minimize on transportation of Ebola patients for long distances. This strategy increases acceptability of the community to bring patients to isolation facilities.

Opinion leaders from affected communities should be engaged to participate in mobilization of their own people to cooperate with the response teams. Psychosocial support should be urgently stepped up targeting the affected communities and families as well as the patients. Ebola response activities should be adapted where necessary to ensure they are more culturally acceptable for the affected communities without compromising on infection prevention and control. Specifically the following recommendations are made:

-Local leaders should take centre stage in directing the Ebola response at the local level, guided by the national and international experts that constitute the outbreak response committees.

- Natives from the affected communities should be trained to conduct house-to-house case search, contact tracing and follow up, and house-to-house health education on Ebola prevention and control.

-Ebola cases should be managed in isolation facilities that are located within the affected communities.

-Health systems should be strengthened through: establishing in-country capacities for laboratory confirmation of Ebola; conducting preparedness trainings on detecting and managing EDPs like Filoviruses and 
enhancing IDSR capacities at all levels; and establishing a community network of community health workers.

-Develop a strategy for enhancing cross-border communications to facilitate follow-up of contacts, case isolation, and educating communities on Ebola prevention and control.

-All political, religious and cultural leaders should pass on messages on Ebola prevention and the need to report suspected cases. Anthropologists and communication experts should be increased (a ratio of 1:50,000 population). All feasible communication channels should be used.

- The affected countries should to vigorously focus on critical sources of infection which include home-based care of patients and burial of affected patients. An ambulance system should collect suspected patients (to the designated isolation health facility) from the community as soon as they are reported by community resource persons. The national response team should train local burial teams who should burry victims. The house or rooms of the dead should be thoroughly disinfected. All beddings, mattresses and clothes that were use by the dead should be burned. If culturally needed, some family members, dressed in appropriate protective wear, should be allowed to participate in or view the preparation of their deceased relatives for burial. Strong messages should be passed to the communities by the political leaders preferably by the Presidents, religious and cultural leaders on the dangers of getting in contact with patients suspected of Ebola and participating in burial of patients without the appropriate personal protective equipment.

-The local administration entities should consider development of by-laws and tough punitive measures that deter the community members from hiding patients, where necessary.

Conclusion

Ebola epidemics have been experienced by several countries in Africa and Uganda has experienced several of them. Several factors highlighted above have been key to the quick containment of Ebola outbreaks in Uganda. West African countries currently experiencing Ebola outbreaks should draw some lessons quickly from the Uganda experience and adapt and adopt them so as to contain the Ebola epidemic. The morbidity and mortality due to the outbreak could be minimized by adopting some of the drivers of success in the Ugandan experience above.

\section{References}

1. World Health Organisation. Ebola virus disease in Guinea, 23 March2014. Retrieved from http://www.afro. who.int/en/clusters-a-programmes/dpc/epidemic-apandemic-alert-and-response/outbreak-news/4063ebola-hemorrhagic-fever-in-guinea. World Health Organisation. 2014a; doi: 10.3201/eid1612.100627.

2. Baize S, Pannetie, D, Oestereich L, Rieger T, Koivogui L, Magassouba N, Gunther, S. Emergence of Zaire Ebola virus disease in Guinea - Preliminary report. The New England Journal of Medicine 2014; doi/ full/10.1056/NEJMoa1404505.

3. Gatherer, D. The 2014 Ebola virus disease outbreak in West Africa. J General Virology.2014; 95:1619-1624. 4. World Health Organisation. Ebola virus disease, Liberia. World Health Organisation. 2014b.

5. World Health Organisation. Ebola virus disease, West Africa. (Update as of 26 May 2014). World Health Organisation. 2014c

6. World Health Organisation. Ebola virus disease, West Africa - Update as of 18 July 2014). World Health Organisation. 2014d

7. Dixon M G, \& Schafer I J. Ebola virial disease outbreak - West Africa, 2014. Morbidity and Mortality Weekly Report, 2014; 63(25): 548-549.

8. Ebola in west Africa: gaining community trust and confidence. Lancet. 2014; 7;383(9933):1946. doi: 10.1016/S0140-6736(14)60938-7

9. Lamunu M, Lutwama J.J, Kamugisha J, Opio A, et al.: Containing a haemorrhagic fever epidemic: the Ebola experience in Uganda (October 2000-January 2001). Int J Infect Dis. 2004, 8: 27-37.

10. Wamala J F, Lukwago L, Malimbo M, Nguku P, Yoti Z, Musenero M, Okware S I. (2010). Ebola Hemorrhagic Fever Associated with Novel Virus Strain, Uganda, 2007-2008. Emerg Infect Dis. 2010.16( 7): 1087-1092. DOI: 10.3201/eid1607.091525.

11. Shoemaker T, MacNeil A, Balinandi S, Campbell S, Wamala J F, McMullan L K, Nichol T. Reemerging Sudan Ebola virus disease in Uganda. Emerg Infect Dis. 2011. 18(9): 1480-1483. DOI: 10.3201/eid1809.111536 12. Centres for Disease Control and Prevention. Ebola Hemorrhagic Fever Outbreak in the Luwero District of Uganda. 2012: Retrieved from http://www.cdc. gov/ncidod/dvrd/spb/outbreaks/index.htm\#ebolaluwero-2012

13. Mbonye A, Wamala J, Winyi-Kaboyo, Tugumizemo V, Aceng J, \& Makumbi I. (2013). Repeated outbreaks of Viral hemorrhagic fevers in Uganda. African Health Sciences. 2013; 12(4): 579-583. 
14. Adjemian J, Farnon E C, Tschioko F, Wamala J F, Byaruhanga E, Bwire G S, Rollin P E. (2011). Outbreak of Marburg hemorrhagic fever among miners in Kamwenge and Ibanda Districts, Uganda, 2007; J Infect Dis. 2011., 204(suppl 3): S796-S799. doi:10.1093/ infdis/jir312.

15. Wamala FJ, Mugagga Malimbo, Okot CL, AtaiOmoruto AD, Tenywa E, Miller JM,.Mbonye AK. (2012). Epidemiological and laboratory characterization of a yellow fever outbreak in northern Uganda, October 2010-January 2011. Int J Infect Dis. 2012. http:// www.ncbi.nlm.nih.gov/pubmed/22575876

16. Francesconi P, Yoti Z, Declich S, Onek PA, Fabiani M, Olango J, Andraghetti R, Rollin PE, Opira C, Greco D, Salmaso S. Ebola hemorrhagic fever transmission and risk factors of contacts, Uganda. Emerg Infect Dis. 2003 Nov;9(11):1430-7.

17. Okware SI, Omaswa FG, Zaramba S, Opio A, et al.: An outbreak of Ebola in Uganda. Trop Med Int
Health. 2002, Vol.7 (Issue 12):1068-1075.

18. Bitekyerezo M, Kyobutungi C, Kizza R, Mugeni J, Munyarugero E, Tirwomwe F, Twongyeirwe E, Muhindo G, Nakibuuka V, Nakate M, John L, Ruiz A, Frame K, Priotto G, Pepper L, Kabakyenga J, Baingana S, Ledo D. The outbreak and control of Ebola viral haemorrhagic fever in a Ugandan medical school. Trop Doct. 2002 Jan; 32(1):10-5.

19. Sandbladh H. Role of the Red Cross movement in Uganda's Ebola outbreak. Bull World Health Organ. 2001; 79(3):267.

20. Anon.: Outbreak of Ebola haemorrhagic fever, Uganda, August 2000-January 2001. Wkly Epidemiol Rec. 2001 Feb 9; 76(6):41-6.

21. MacNeil A, Farnon EC, Wamala J, Okware S, Cannon DL, Reed Z, Towner JS, Tappero JW, Lutwama J, Downing R, Nichol ST, Ksiazek TG, Rollin PE. Proportion of deaths and clinical features in Bundibugyo Ebola virus infection, Uganda. Emerg Infect Dis. 2010 Dec;16(12):1969-73. 